\title{
Research Paper: Serum Eicosapentaenoic and Arachidonic Acids Concentration and Cognitive Function in Patients With Coronary Artery Disease
}

\author{
1. Neurosciences Research Center, Qom University of Medical Sciences, Qom, Iran. \\ 2. Spiritual Health Research Center, Qom University of Medical Sciences, Qom, Iran. \\ 3. Student Research Committee, Qom University of Medical Sciences, Qom, Iran. \\ 4. Research Center for Environmental Pollutants, Qom University of Medical Sciences, Qom, Iran. \\ 5. Department of Cardiology, Faculty of Medicine, Qom University of Medical Sciences, Qom, Iran.
}

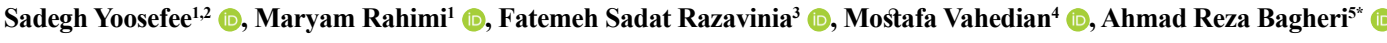

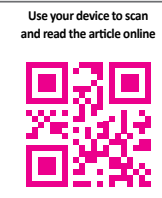

Citation: Yoosefee, S., Rahimi, M., Razavinia, F. S., Vahedian, M., \& Bagheri, A. R. (2020). Serum Eicosapentaenoic and Arachidonic Acids Concentration and Cognitive Function in Patients With Coronary Artery Disease. Basic and Clinical Neuroscience, 12(1), 63-68. http://dx.doi.org/10.32598/bcn.12.1.606.3

doi) $h$ http://dx.doi.org/10.32598/bcn.12.1.606.3

\section{(c) (1) (8)}

Article info:

Received: 16 Apr 2020

First Revision: 22 May 2020

Accepted: 19 Jul 2020

Available Online: 01 Jan 2021

Keywords:

Eicosapentaenoic acid, Arachidonic acid, Cognitive function, Mini Mental State Examination, Montreal cognitive assessment, Coronary artery disease

\section{A B S T RA C T}

Introduction: Recent studies have shown that Polyunsaturated Fatty Acids (PUFAs), including Eicosapentaenoic Acid (EPA), and Arachidonic Acid (AA), are associated with cognitive functions in patients with Coronary Artery Disease (CAD). Nevertheless, controversial results have been reported, too. The current study aimed to clarify the association of serum EPA and AA levels with cognitive decline in an Iranian sample with CAD.

Methods: We evaluated cognitive function with the Mini-Mental State Examination (MMSE) and Montreal Cognitive Assessment (MoCA), in relation to serum levels of EPA and AA, in 179 CAD patients. The associations between the MMSE and MoCA scores and the other demographic parameters were evaluated.

Results: Patients with CAD generally had mild cognitive impairment. But we could not find any significant correlation between PUFAs and cognitive function. However, BMI was associated with EPA/MoCA; age was associated with MMSE/MoCA and BMI. Finally, the correlation between sex and MMSE/MoCA was significant.

Conclusion: Subjects generally had mild cognitive impairment, but we could not find any significant correlation between serum EPA and AA levels with cognitive function.

\section{* Corresponding Author:}

Ahmad Reza Bagheri, MD.

Address: Department of Cardiology, Faculty of Medicine, Qom University of Medical Sciences, Qom, Iran.

Tel: +98 (25) 36122156

E-mail:drarbagheri@yahoo.com 


\section{Highlights}

- We could not find any significant correlation between eicosapentaenoic acid and arachidonic acid with cognitive impairment in patients with coronary artery disease.

- In consistent with other studies, we found that patients with coronary artery disease generally had mild cognitive impairment.

- To confirm the findings of this study, further studies are needed with large sample sizes in diverse population.

\section{Plain Language Summary}

One of the problems of patients with coronary artery disease is cognitive dysfunction. This causes problems in these patients, including medication management and self-care. Recently, some studies have shown that intake of polyunsaturated fatty acids, including eicosapentaenoic acid and arachidonic acid, are associated with a reduced risk of cognitive dysfunction in these patients. However, controversial results have also been reported. The aim of this study was to investigate the relationship between serum levels of these two polyunsaturated fatty acids and cognitive functions in patients with coronary artery disease in an Iranian population. Although our findings indicate a mild cognitive impairment in these patients, we did not find any significant relationship between the studied fatty acids and cognitive function. However, the results of this study should be further investigated in additional studies with larger sample sizes.

\section{Introduction}

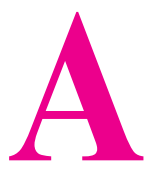

s confirmed by evidence from different populations, cognitive impairment is not only the main characteristic of diseases such as schizophrenia (Aleman, Hijman, De Haan, \& Kahn, 1999; Ananloo, Yoosefee, \& Karimipour, 2020; Hatami et al., 2017; Hegde et al., 2013; Toulopoulou, Morris, RabeHesketh, \& Murray, 2003), but also an essential factor in cardiovascular diseases, though the latter receiving less attention (Kovacic, Castellano, \& Fuster, 2012; Schmidt et al., 2008). Cardiac dysfunction leads to cerebral blood flow problems and may be associated with cognitive impairments. Conversely, cognitive impairment could be an independent cardiovascular risk factor (O'Donnell et al., 2012). A recent study reports the prevalence of $\operatorname{cog}$ nitive impairments in patients with Coronary Artery Disease (CAD), higher than previously reported (Eftekhari et al., 2018). Eicosapentaenoic Acid (EPA) is an n-3 Polyunsaturated Fatty Acid (PUFA) that may be responsible for fish oil benefits. Studies have shown that intake of EPA is associated with reduced risk of cognitive impairment (Morris et al., 2003; Yagi et al., 2014) and CAD (Yokoyama et al., 2007). Contrary to n-3 PUFA, a high concentration of Arachidonic Acid (AA), an n-6 PUFA, and a low ratio of serum EPA to AA (EPA/AA ratio) are associated with major adverse cardiac events (Matsuzaki et al., 2009; Ueeda et al., 2011). However, the results of cross-sectional and longitudinal studies examining the association between EPA and AA with cognitive performance are inconsistent, with some studies showing association (Kalmijn et al., 2004; Tokuda et al., 2014), and other studies showing no association (van de Rest et al., 2009; Yagi et al., 2014). Therefore, the current study aimed to explore the association between cognitive function and EPA and AA in a sample of Iranian CAD patients.

\section{Materials and Methods}

\subsection{Patients and study design}

The study population consisted of 179 patients with CAD referred to the Cardiology Ward at Shahid Beheshti University Hospital in Qom, a city in the central region of Iran, between March and October 2018. Serum EPA and AA levels of the participants were measured. Besides, the patients underwent Mini-Mental State Examinations (MMSE) and Montreal Cognitive Assessment (MoCA) to screen their cognitive function. The inclusion criteria were a history of myocardial infarction, angiographic evidence of at least $50 \%$ stenosis in at least 1 coronary artery, evidence of exercise-induced ischemia, or history of coronary revascularization. Those with a history of myocardial infarction in the last month, patients with neurocognitive problems documented in the medical record, substance abuse within the past 5 years, symptomatic active malignant or liver diseases, and the patients who had used fish oil supplements or fatty acid- 
containing drugs, were excluded from the study. Moreover, patients unable to fill out the questionnaires did not meet the inclusion criteria.

The study population filled up a demographic parameters questionnaire, including age, gender, education, and Body Mass Index (BMI) (which was calculated). Furthermore, their serum fatty acid composition, including levels of EPA and AA, were measured by gas-liquid chromatography at the hospital laboratory. Cognitive function was evaluated by MMSE, which is a widely-used screening tool for the assessment of cognitive function (Folstein, Folstein, \& McHugh, 1975) and MoCA (Nasreddine et al., 2005).

\subsection{Statistical analysis}

Descriptive analyses were expressed as the Mean \pm SD. The Pearson correlation analyzed the correlations between variables. All statistical analyses were performed in SPSS V. 22. Statistical significance was defined as $\mathrm{P}<0.05$.

\section{Results}

The patients' demographic characteristics are presented in Table 1. The Mean \pm SD MMSE and MoCA scores were 22.64 (4.77) and 17.06 (5.82), respectively, indicating that participants generally had mild cognitive impairment (Eftekhari et al., 2018).

As shown in Table 2, we could not find any significant correlation between EPA and AA with cognitive impairment (both MMSE and MoCA scores) $(\mathrm{P}>0.05)$. However, considering other variables, the correlation between $\mathrm{BMI}$ and EPA/MoCA was significant $(\mathrm{P}<0.05)$. The correlation between age and MMSE/MoCA was significant too $(\mathrm{P}<0.001)$, and the correlation between sex and MMSE/MoCA was significant as well $(\mathrm{P}<0.001)$

\section{Discussion}

The participants of the present study generally had mild cognitive impairment. However, the study did not reveal any significant correlation between serum levels

Table 1. Demographic characteristics of participants

\begin{tabular}{ccccc}
\hline Variable & No. & Min. & Max. & Mean \pm SD \\
\hline Age & 179 & 32 & 85 & $58.99 \pm 10.07$ \\
BMI & 179 & 18.78 & 47.95 & $28.26 \pm 4.11$ \\
\hline A.A & 179 & 0.80 & 179 & $17.03 \pm 5.97$ \\
\hline EPA & 179 & 1 & 2281 & $157.60 \pm 33.45$ \\
MMSE & 179 & 10 & 30 & $22.64 \pm 4.77$ \\
MoCA & 179 & 3 & 29 & $17.06 \pm 5.82$ \\
\hline
\end{tabular}

Table 2. Correlation among PUFAs, MMSE/MoCA scores and demographic charactesistics

\begin{tabular}{|c|c|c|c|c|c|c|c|c|c|}
\hline Variable & EPA & A.A & MMSE & MoCA & Age & BMI & Sex & DM & Education \\
\hline EPA & 1 & & & & & & & & \\
\hline A.A & $0.93 * *$ & 1 & & & & & & & \\
\hline MMSE & 0.02 & -0.02 & 1 & & & & & & \\
\hline MoCA & 0.07 & 0.05 & $0.86 * *$ & 1 & & & & & \\
\hline Age & -0.08 & -0.05 & $-0.28 * *$ & $-0.37^{* *}$ & 1 & & & & \\
\hline $\mathrm{BMI}$ & $-0.15^{*}$ & -0.14 & 0.12 & $.15^{*}$ & $-0.20 * *$ & 1 & & & \\
\hline Sex & -0.01 & -0.03 & $0.39 * *$ & $0.34 * *$ & 0.06 & $-0.17^{*}$ & 1 & & \\
\hline DM & -0.03 & 0.02 & -0.07 & -0.04 & 0.10 & 0.13 & $-0.16^{*}$ & 1 & \\
\hline Education & 0.10 & 0.10 & $0.64 * *$ & $0.64 * *$ & $-0.30 * *$ & 0.02 & $0.37 * *$ & -0.13 & 1 \\
\hline
\end{tabular}

*Significant $(\mathrm{P}<05)$; **Significant $(\mathrm{P}<0001)$.

NEUR SCIENCE 
of EPA and AA with cognitive impairment. Moreover, the results indicated that BMI was significantly associated with EPA/MoCA; age significantly was associated with MMSE/ MoCA, and the correlation between sex and MMSE/MoCA was significant, too. Some studies have confirmed the influence of $\mathrm{CAD}$ and its risk factors upon the incidence of degenerative brain pathologies and consequent cognitive impairment (Abete et al., 2014; Eftekhari et al., 2018), which are consistent with the results of this study. Another study showed that serum EPA concentration was associated with cognitive function in patients with CAD (Yagi et al., 2014) and middle age (Kalmijn et al., 2004).

On the other hand, a few small studies have reported the benefits of EPA in people with confirmed dementia (Boston, Bennett, Horrobin, \& Bennett, 2004). These results are challenged by the findings of longitudinal analyses, over 6 years of follow-up by van de Rest and colleagues. They did not show any significant associations between EPA intake and cognitive change (van de Rest et al., 2009). This result is in accordance with our findings that support no association between PEA and cognitive function.

Regarding AA, although there are few studies consistent with this study, Yagi et al. (2014) found no relationship between MMSE and AA in patients with CAD (Yagi et al., 2014). On the other hand, Tokuda et al (2014)' findings suggest that lower serum AA levels were associated with cognitive function in older men (Tokuda et al., 2014). Another study showed that the serum levels of dihomo$\gamma$-linolenic acid (DGLA) and n-6 PUFA were associated with cognitive function in patients with CAD (Ishihara et al., 2019). It seems that further studies are needed with big enough sample sizes within diverse races.

\section{Conclusion}

Although subjects with CAD generally had mild cognitive impairment, no significant correlation is found between serum EPA and AA levels with cognitive function.

\section{Ethical Considerations}

\section{Compliance with ethical guidelines}

Researchers took into account the ethical considerations, including institutional and national standards. Every patient received a full explanation of the procedures and was requested to fill up an informed consent form. In every stage of the research, the patients were allowed to withdraw if they wished. This study was approved by the Qom University of Medical Sciences Ethics Committee.

Funding

This work was funded by Qom University of Medical Sciences.

\section{Authors' contributions}

Concept, design, and supervision: Sadegh Yoosefee, Ahmad Reza Bagheri; Funding: Ahmad Reza Bagheri; Data collection and processing: Sadegh Yoosefee, Maryam Rahimi, Fatemeh Sadat Razavinia, Ahmad Reza Bagheri; Analysis: Mostafa Vahedian; Literature search: Sadegh Yoosefee, Maryam Rahimi, Fatemeh Sadat Razavinia, Mostafa Vahedian, Ahmad Reza Bagheri; Writing: Sadegh Yoosefee, Ahmad Reza Bagheri; Critical review: All Authors.

\section{Conflict of interest}

The authors declared no conflict of interest.

\section{Acknowledgments}

We would like to express our gratitude to the Department of Cardiology, Qom University of Medical Sciences, and all participants.

\section{References}

Abete, P., Della-Morte, D., Gargiulo, G., Basile, C., Langellotto, A., \& Galizia, G., et al. (2014). Cognitive impairment and cardiovascular diseases in the elderly. A heart-brain continuum hypothesis. Ageing Research Reviews, 18, 41-52. [DOI:10.1016/j. arr.2014.07.003] [PMID]

Aleman, A., Hijman, R., De Haan, E. H., \& Kahn, R. S. (1999) Memory impairment in schizophrenia: A meta-analysis. American Journal of Psychiatry, 156(9), 1358-66. https://ajp. psychiatryonline.org/doi/full/10.1176/ajp.156.9.1358

Ananloo, E., Yoosefee, S., \& Karimipour, M. (2020). Neuregulin1 gene variants as a biomarker for cognitive impairments in patients with schizophrenia. The European Journal of Psychiatry, 34(1), 11-9. [DOI:10.1016/j.ejpsy.2019.12.004]

Boston, P. F., Bennett, A., Horrobin, D. F., \& Bennett, C. N. (2004). Ethyl-EPA in Alzheimer's disease-a pilot study. Prostaglandins, Leukotrienes and Essential Fatty Acids (PLEFA), 71(5), 341-6. [DOI:10.1016/j.plefa.2004.07.001] [PMID]

Eftekhari, S. S., Hejazi, S. A., Sharifipour, E., Hejazi, S. F., Talebizadeh, M., \& Mostafavi, H., et al. (2018). Cognitive impairment in patients with coronary artery disease; comparison of Montreal Cognitive Assessment (MoCA) and Mini Men- 
tal State Examination (MMSE).Journal of Advances in Medical and Biomedical Research, 26(119), 12-6. [DOI:10.30699/ jambs.26.119.12]

Folstein, M. F., Folstein, S. E., \& McHugh, P. R. (1975). Minimental state: A practical method for grading the cognitive state of patients for the clinician. Journal of Psychiatric Research, 12(3), 189-98. [DOI:10.1016/0022-3956(75)90026-6]

Hatami, M., Karamghadiri, N., Mohaghegh, H., Yoosefee, S., Karimipoor, M., \& Hadjighasem, M., et al. (2017). Neuregulin-1 gene and schizophrenia, and its negative symptoms in an Iranian population. Iranian Journal of Psychiatry and Behavioral Sciences, 11(4). [DOI:10.17795/ijpbs-4484]

Hegde, S., Thirthalli, J., Rao, S. L., Raguram, A., Philip, M., \& Gangadhar, B. (2013). Cognitive deficits and its relation with psychopathology and global functioning in first episode schizophrenia. Asian Journal of Psychiatry, 6(6), 537-43. [DOI:10.1016/j.ajp.2013.07.002] [PMID]

Ishihara, K., Izawa, K. P., Kitamura, M., Shimogai, T., Kanejima, Y., \& Morisawa, T., et al. (2019). Serum concentration of dihomo- $\gamma$-linolenic acid is associated with cognitive function and mild cognitive impairment in coronary artery disease patients. Prostaglandins, Leukotrienes and Essential Fatty Acids, 158, 102038. [DOI:10.1016/j.plefa.2019.102038] [PMID]

Kalmijn, S., Van Boxtel, M., Ocke, M., Verschuren, W., Kromhout, D., \& Launer, L. (2004). Dietary intake of fatty acids and fish in relation to cognitive performance at middle age. Neurology, 62(2), 275-80. [DOI:10.1212/01.WNL.0000103860.75218. A5] [PMID]

Kovacic, J. C., Castellano, J. M., \& Fuster, V. (2012). The links between complex coronary disease, cerebrovascular disease, and degenerative brain disease. Annals of the New York Academy of Sciences, 1254(1), 99-105. [DOI:10.1111/j.17496632.2012.06482.x] [PMID]

Matsuzaki, M., Yokoyama, M., Saito, Y., Origasa, H., Ishikawa, Y., \& Oikawa, S., et al. (2009). Incremental effects of eicosapentaenoic acid on cardiovascular events in statin-treated patients with coronary artery disease. Circulation Journal, 73(7), 1283-90. [DOI:10.1253/circj.CJ-08-1197] [PMID]

Morris, M. C., Evans, D. A., Bienias, J. L., Tangney, C. C., Bennett, D. A., \& Wilson, R. S., et al (2003). Consumption of fish and n-3 fatty acids and risk of incident Alzheimer disease. Archives of neurology, 60(7), 940-6. [DOI:10.1001/archneur.60.7.940] [PMID]

Nasreddine, Z. S., Phillips, N. A., Bédirian, V., Charbonneau, S., Whitehead, V., \& Collin, I., et al. (2005). The Montreal Cognitive Assessment, MoCA: A brief screening tool for mild cognitive impairment. Journal of the American Geriatrics Society, 53(4), 695-9. [DOI:10.1111/j.1532-5415.2005.53221.x] [PMID]

O’Donnell, M., Teo, K., Gao, P., Anderson, C., Sleight, P., \& Dans, A., et al. (2012). Cognitive impairment and risk of cardiovascular events and mortality. European Heart Journal, 33(14), 1777-86. [DOI:10.1093/eurheartj/ehs053] [PMID]

Schmidt, S. L., Correa, P. L., Tolentino, J. C., Manhães, A. C., Felix, R. M., \& Azevedo, J. C., et al. (2008). Value of combining activated brain FDG-PET and cardiac MIBG for the differential diagnosis of dementia: differentiation of dementia with Lewy bodies and Alzheimer disease when the diagnoses based on clinical and neuroimaging criteria are difficult. Clinical Nuclear Medicine, 33(6), 398-401. [DOI:10.1097/ RLU.0b013e3181708244] [PMID]
Tokuda, H., Kontani, M., Kawashima, H., Akimoto, K., Kusumoto, A., \& Kiso, Y., et al. (2014). Arachidonic acid-enriched triacylglycerol improves cognitive function in elderly with low serum levels of arachidonic acid. Journal of Oleo Science, 63(3), 219-27. [DOI:10.5650/jos.ess13195] [PMID]

Toulopoulou, T., Morris, R. G., Rabe-Hesketh, S., \& Murray, R. M. (2003). Selectivity of verbal memory deficit in schizophrenic patients and their relatives. American Journal of Medical Genetics Part B: Neuropsychiatric Genetics, 116(1), 1-7. [DOI:10.1002/ajmg.b.10027] [PMID]

Ueeda, M., Doumei, T., Takaya, Y., Ohnishi, N., Takaishi, A., \& Hirohata, S., et al. (2011). Association of serum levels of arachidonic acid and eicosapentaenoic acid with prevalence of major adverse cardiac events after acute myocardial infarction. Heart and Vessels, 26(2), 145-52. [DOI:10.1007/s00380010-0038-8] [PMID]

van de Rest, O., Spiro III, A., Krall-Kaye, E., Geleijnse, J. M., de Groot, L. C., \& Tucker, K. L. (2009). Intakes of (n-3) fatty acids and fatty fish are not associated with cognitive performance and 6-year cognitive change in men participating in the veterans affairs normative aging study. The Journal of Nutrition, 139(12), 2329-36. [DOI:10.3945/jn.109.113647] [PMID] [PMCID]

Yagi, S., Hara, T., Ueno, R., Aihara, K.-i., Fukuda, D., \& Takashima, A., et al. (2014). Serum concentration of eicosapentaenoic acid is associated with cognitive function in patients with coronary artery disease. Nutrition Journal, 13(1), 112. [DOI:10.1186/1475-2891-13-112] [PMID] [PMCID]

Yokoyama, M., Origasa, H., Matsuzaki, M., Matsuzawa, Y., Saito, Y.,\& Ishikawa, Y., et al. (2007). Effects of eicosapentaenoic acid on major coronary events in hypercholesterolaemic patients (JELIS): A randomised open-label, blinded endpoint analysis. The Lancet, 369(9567), 1090-8. [DOI:10.1016/S01406736(07)60527-3] 
This Page Intentionally Left Blank 